Final Report

to

The Department of Energy

for a program of research entitled:

\title{
MEASUREMENTS OF NANOSTRUCTURES WITH MICROMACHINED MICROSCOPES
}

\author{
Principal Investigators: \\ Gordon. S. Kino, Electrical Engineering and William E. Moerner, Chemistry
}

Grant No. DE-FG03-00ER45815

April 2005

Edward L. Ginzton Laboratory

450 Via Palou, Stanford University

Stanford, CA 94305-4085

Tel: 650-723-0205, Fax: 650-494-7018, EM: kino@stanford.edu 


\title{
MEASUREMENTS OF NANOSTRUCTURES WITH MICROMACHINED MICROSCOPES
}

\author{
Gordon. S. Kino and William E. Moerner
}

\begin{abstract}
Summary
Semiconductor devices, optical storage devices, and hard disks have minimum feature sizes that are becoming smaller and smaller. The minimum feature size in production devices is presently less than $130 \mathrm{~nm}$, and far smaller devices are forecast for the future. As each generation of devices decreases in size, it is often said that the familiar optical techniques employed for measurement and lithography will no longer be viable. However, the use of near-field principles - in which definition is no longer limited by diffraction—makes it possible to observe feature sizes considerably smaller than $100 \mathrm{~nm}$.
\end{abstract}

The work carried out in the first two years of this program used a micromachined solid immersion lens (SIL) of a few microns in diameter. Using a silicon SIL, we reduced the diffraction limited spot size for infrared imaging to obtain a $1.9 \mu \mathrm{m}$ spot size at a wavelength $\mathrm{l}=9.3 \mu \mathrm{m}(\mathrm{l} / 5)$ and $128 \mathrm{~nm}$. Then, in an associated program with Professor C.F. Quate, we obtained spot sizes of $128 \mathrm{~nm}$ at $405 \mathrm{~nm}$ wavelength in the visible range. These lenses were mounted on cantilevers similar to those used in atomic force microscopes (AFM). The metal antennas made in our associated program were resonant in the wavelength range from 1-10 $\mu \mathrm{m}$. This work was described in a previous progress report and in published papers.

During the past three years, we have developed optical techniques for nanostructures with definitions in the sub $30 \mathrm{~nm}$ range. We have created metal nanostructures for single photon and two-photon near-field microscopy, and for Raman spectroscopy. Raman spectroscopy is important because it permits identification of organic materials in small regions without using stain or fluorescent dyes. We are also interested in scanned reflection microscope imaging with definitions in the 20-30 nm size range, in one and two-photon lithography with similar definitions, and in using Raman excitation for measuring and identifying materials down to the size of single molecules.

The work was carried out by Professor G.S. Kino (Electrical Engineering) and Professor W.E. Moerner (Chemistry). The early work on solid immersion lenses was carried out in cooperation with Professor K.E. Goodson (Mechanical Engineering). Kino’s expertise is in scanning optical microscopy and micromachining; Moerner is an expert on single molecule interactions and Raman spectroscopy.

In the past, laser excitation of plasmon resonances in silver and gold particles has been used to generate high fields in a small area in the neighborhood of small particles. Relatively high power excitation of the fields in the neighborhood of a sharp tip-typically a metal-coated AFM tip-has also been used to generate high fields for Raman excitation. In some cases, outstanding definition in the required range has been obtained. However, because these techniques are not easily reproducible with tailored characteristics, they are not easy to use in actual practice. We have engineered reproducible resonant bowtie antenna structures that can excite extremely intense fields in a small region of 10-30 nm. 
We have:

1. Made reproducible scanning probes with high efficiency, and predictable and reproducible characteristics. We obtained good efficiency with dimensions well below the diffraction limit, so that relatively small laser powers in the milliwatt range can be used. For single frequency operation, only low power is necessary to obtain very high fields for the excitation of well-defined Raman scattering, and to work in a reflection mode with good scanning speeds;

2. Obtained predictable results with very high fields suitable for obtaining Raman scattering and twophoton scattering;

3. Made a scanning probe mounted on a micromachined cantilever to obtain high definition reflection mode images that can be scanned rapidly;

4. Observed Raman scattering using bowtie antennas with CW excitation.

\section{Introduction}

When light of wavelength $\lambda$ is focused into a medium of refractive index $n$, the minimum spot size due to diffraction is of the order of $\lambda /(2 n)$. For example, at a wavelength of $405 \mathrm{~nm}$, diffraction limits the minimum spot size to be greater than $200 \mathrm{~nm}$. Near-field techniques, based on the idea that light can be passed through a tapered metal-covered optical fiber that acts as a cut-off waveguide when the guide diameter is less than $\lambda /(2 n)$, have made it possible to obtain spot sizes of the order of $50 \mathrm{~nm}$, but with power transmission on the order of $10^{-3}$ to $10^{-6}$ of the incident power ${ }^{1}$. Similarly, passing light through a small pinhole on the order of $50 \mathrm{~nm}$ diameter leads to a field intensity at the end of the guide that is greatly reduced from that of the incident field.

An alternative technique, “Apertureless Imaging,” illuminates nanoparticles or sharp tips (like atomic force microscope probes) with an incident beam ${ }^{2,3}$. Because of the so-called "lightning rod" effect, the field intensity in nanosized subwavelength regions near the sharp edges of metal nanoparticles or sharp tips can be much greater than the intensity of the incident wave. Antenna-like resonances or plasmon resonances can further enhance the fields. These effects can be used for a variety of applications such as near-field imaging ${ }^{4}$ and Raman spectroscopy ${ }^{5}$, and for guiding electromagnetic energy in subwavelength-sized optoelectronic devices ${ }^{6}$.

Crozier et al. ${ }^{7}$ studied metallic antennas at mid-infrared wavelengths with shapes and sizes determined by electron-beam lithography, and explored the reasons for electric field enhancement by computing the current distribution in the antennas. They found good agreement between the experimental extinction efficiency and resonant wavelengths, and the values obtained from finite difference time domain (FDTD) computations. At infrared wavelengths, the resonant length of these antennas along the direction of incident polarization is typically about $\lambda /\left(2 n_{s}\right)$ where $n_{s}$ is the refractive index of the substrate on which the metal is deposited. However, in the optical range, as we shall show, the resonance for antennas made from $\mathrm{Au}$ and $\mathrm{Ag}$ is highly dependent on the plasmon resonant frequency, and the required size for resonance is much smaller than $\lambda /\left(2 n_{s}\right)$.

Many groups have studied nanoparticles of various shapes to enhance field intensity, and have used various theoretical techniques to predict behavior. Genov et al. ${ }^{8}$ studied gold nanodisks separated by small gaps, and predicted near-field intensity enhancements greater than $10^{3}$. Hao et al. ${ }^{9}$ used the discrete dipole approximation method to study nanostructures of different shapes and sizes, including two triangu- 
lar nanoprisms facing each other tip-to-tip and separated by $2 \mathrm{~nm}$, and predicted intensity enhancements as high as $\sim 5 \times 10^{4}$. Rechberger et al. ${ }^{10}$ experimented with coupling between gold nano disks, and showed that the resonant wavelength depended on the spacing between the particles.

\section{Bowtie Antennas}
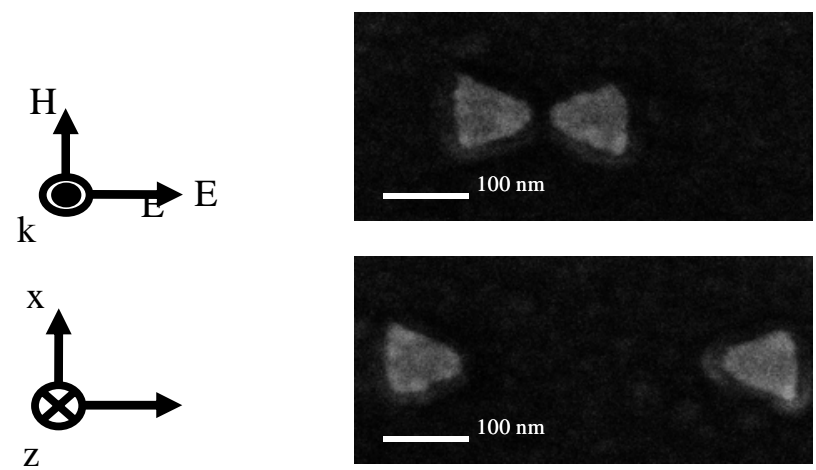

Figure 1: SEM images of two representative gold bowties on a fused silica-ITO substrate. The polarization of the incident beam and the co-ordinate system are shown.

Grober et al. ${ }^{11}$ suggested that a receiving antenna shaped like a bowtie could be used to receive an incident wave with its E-field in the $y$-direction, along the line between the two opposing triangles (see Fig. 1 for an example of bowties fabricated to resonate at optical wavelengths.) This design produces a large field between the facing tips of the two triangles comprising the bowtie. They demonstrated this principle at microwave frequencies, and suggested extending its use to visible wavelengths. Following this suggestion, we have fabricated and experimentally characterized metallic Au bowtie structures of sufficiently small size to resonate at visible/near-ir wavelengths ${ }^{12}$.

Our experiments were designed to make reproducible sub-100 nm sized bowties that could be located at specified positions. To achieve this aim, Au bowties were fabricated at the Stanford Nanofabrication Facility with electron beam lithography (EBL) on a transparent substrate, using a commercial EBL tool (Raith 150). Substrates consisted of a 160- $\mu$ m thick fused silica coverslip (refractive index, $n=$ 1.47), overcoated with $50 \mathrm{~nm}$ of indium tin oxide (ITO) to reduce charging effects during EBL. Goodberlet et al. ${ }^{13}$ demonstrated the ability to write line widths of $19 \mathrm{~nm}$ with this tool using single pass lines. The bowties were laid out using single pass lines to reduce proximity effects, thus increasing EBL resolution. Prior to EBL writing, the sample was cleaned in acetone and baked in an oven at $150^{\circ} \mathrm{C}$ for 2 hrs to remove moisture. A $50 \mathrm{~nm}$-thick layer of poly-methyl-methacrylate (PMMA) (1\% in chlorobenzene, 950,000 m.w.) was spun on the ITO layer, and the sample was baked in an oven at $150^{\circ} \mathrm{C}$ for 2 hours to harden the resist and remove residual solvent. During EBL, the resist was patterned at an acceleration voltage of $10 \mathrm{kV}$ and a dose of $275 \mathrm{pC} / \mathrm{cm}$. After exposure, the resist was developed at $22^{0} \mathrm{C}$ for 28 seconds, using a 25\% methyl-iso-butyl-ketone in isopropyl alcohol solution. A $5 \mathrm{~nm}$ Cr adhesion layer and $20 \mathrm{~nm}$ Au were then deposited by an electron-beam evaporator, and the patterns were transferred to the substrate via a lift-off process.

Scanning electron microscope (SEM) images of representative bowties are shown in Figure 1. For this study, the shape of each constituent triangle of a bowtie was held constant at $75 \mathrm{~nm}$ in length (the distance from the midpoint of the triangle base to its apex); gap lengths were varied from $16 \mathrm{~nm}$ to nearly $500 \mathrm{~nm}$; and the triangular tips were observed to have a radius of curvature of $16 \mathrm{~nm}$. Single bowties 
were separated by $10-\mu \mathrm{m}$ pitch to eliminate long-range coupling effects, and to ensure that only scattering from a single bowtie was collected ${ }^{12}$.

\subsection{Single-Photon Effects}

Single bowtie scattering spectra were measured with far-field total internal reflection (TIR) microscopy. Using this method, the excitation beam is trapped in the evanescent field until scattered toward the detector by the bowtie. Broadband light from a 30W halogen lamp was passed through a $455 \mathrm{~nm}$ long-pass filter, and coupled into a multimode fiber (65 $\mu \mathrm{m}$ core) that served as a spatial filter. The fiber output was collimated, and focused into a fused silica prism. Index-matching fluid (refractive index $n=$ 1.47) coupled the prism to the bottom of the fused silica substrate, placing the Au bowties at the ITO-air interface that was also the location of the evanescent field. The excitation light was s-polarized, and the axis of each bowtie was carefully oriented parallel to the polarization axis. (Perpendicular polarization leads to unremarkable results, similar to the scattering from isolated triangles ${ }^{12}$.)

Scattered light was collected with an air objective (100X, 0.8 numerical aperture, NA); a 50 $\mu$ m pinhole was placed at the microscope image plane to spatially filter the collected light to an area $\sim 0.5 \mu \mathrm{m}$ in diameter on the sample plane. The antennas were located by scanning the sample stage and collecting broadband scattered light with a single photon-counting avalanche photodiode. Spectra were acquired during $60 \mathrm{~s}$ exposures by dispersing the scattered light with a 150 lines/mm grating spectrograph, with a liquid-nitrogen-cooled charged coupled device Si detector at the image plane. All spectra were corrected for the wavelength and polarization dependences of the lamp emission, the optical system throughput, and the detector quantum efficiency. The measured results were compared to finite-difference time-domain FDTD calculations of the electromagnetic field distributions.

The electric field variation in the vicinity of a bowtie antenna was determined theoretically through 3-dimensional FDTD simulations ${ }^{14}$ (and experimentally by the use of two-photon scattering effects described below). The FDTD simulations did not make any approximations to compute the final field intensity in the vicinity of the bowties. The size of each node in the simulation grid is $4 \mathrm{~nm}$ along the $\mathrm{x}, \mathrm{y}$, and $\mathrm{z}$ directions. In the simulations, the bowtie antennas are illuminated from the substrate side by a plane wave polarized along the y-direction, and the antennas radiate into free space. The wavelengthdependent dielectric constants used for the bowtie antennas and the substrate are taken from Palik et al. ${ }^{15}$. Perfectly matched layers (PML) are used at the top and bottom of the simulation domain to completely absorb waves, leaving the simulation domain in the direction of propagation. The FDTD simulations assume periodic boundary conditions in the $x-y$ directions, thereby simulating an array of antennas; however, the experimental results were obtained for individual bowtie antennas. The inter-element spacing between the antennas in the FDTD simulation was chosen to be sufficiently large that coupling between the antennas had a negligible effect on the near-field distribution in each antenna. This method enabled a direct comparison between theory and experiment.

All the bowtie antennas simulated have constituent triangles that are equilateral, where each side is $88 \mathrm{~nm}$ in length, with tip-to-base length $75 \mathrm{~nm}$, and the radius of curvature at the triangle apex equal to $12 \mathrm{~nm}$. The gap between the triangles in the bowtie was varied from $16 \mathrm{~nm}$ to $500 \mathrm{~nm}$. The thickness of each bowtie antenna simulated was $24 \mathrm{~nm}$ ( $20 \mathrm{~nm}$ gold layer $+4 \mathrm{~nm}$ chrome sticking layer). The substrate was fused silica ( $n=1.47$ ) with a $52 \mathrm{~nm}$ layer of indium tin oxide (ITO, $n=1.95$ ) evaporated on it. These values are very close to specifications of the fabricated bowties ${ }^{12,16}$. The results of the calculations for field intensity and scattering efficiency are shown in Fig. 2. 


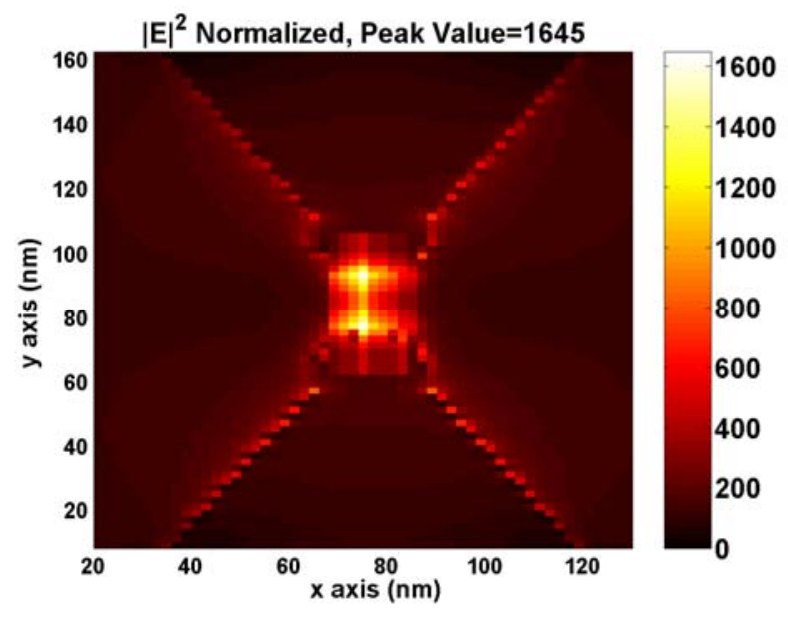

(a)

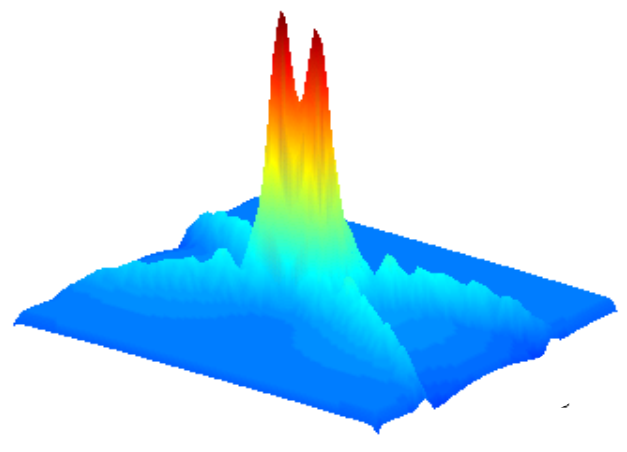

(b)

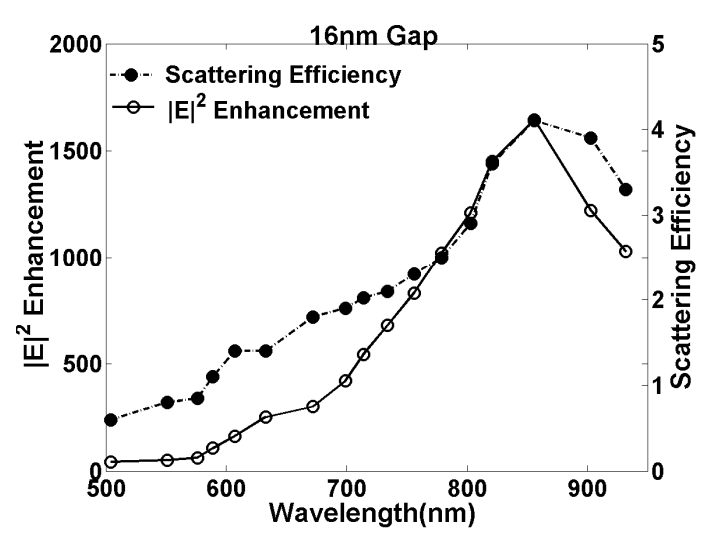

(c)

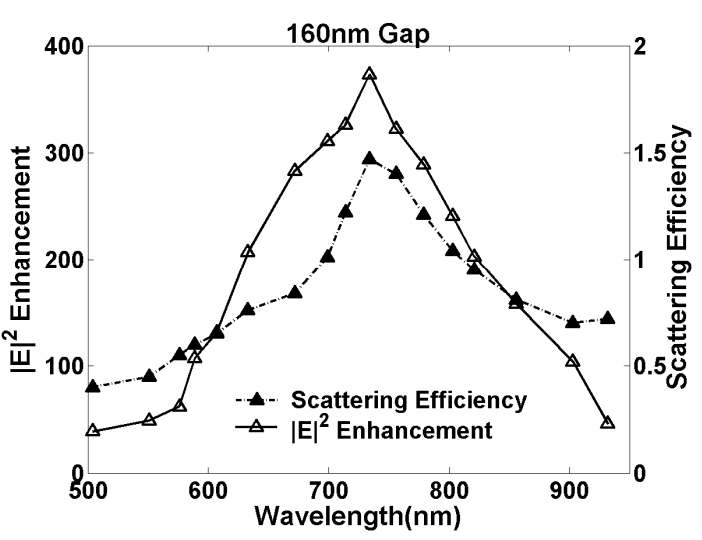

(d)

Figure 2(a) FDTD calculations of $|\mathrm{E}|^{2}$ enhancement $4 \mathrm{~nm}$ above the top surface of a $16 \mathrm{~nm}$ gap bowtie antenna; (b) A 3-D surface plot of the peak intensity variation across the bowtie is also shown, with a different color scale. The illumination is from the substrate side and the illumination wavelength is $856 \mathrm{~nm}$. The peak value of intensity in both plots is 1645 times that of the incident field. The maximum intensity value, $4 \mathrm{~nm}$ above the bowtie surface, is close to each triangle apex with a slight dip in the middle of the gap ${ }^{6}$; (c) is a plot of the maximum $|\mathrm{E}|^{2}$ enhancement and a plot of the scattering efficiency as a function of wavelength for a $16 \mathrm{~nm}$ gap; (d) are similar plots to (c) for a $160 \mathrm{~nm}$ gap. The bowtie with $160 \mathrm{~nm}$ gap has a far smaller enhancement, and peaks at $730 \mathrm{~nm}$ wavelength. The direction of the maximum field for gaps smaller than $60 \mathrm{~nm}$ is along the plane of the bowties in the y-direction; for gaps greater than $60 \mathrm{~nm}$, maximum field for gaps is in the direction of propagation (z-direction), perpendicular to the surface of the metal ${ }^{1}$ With gap spacing smaller than $16 \mathrm{~nm}$ and tip radii of curvature smaller than $12 \mathrm{~nm}$, we have observed stronger fields, both experimentally and by calculation. However, smaller and well-controlled gap spacings and tip radii are difficult to reliably fabricate in practice

The experimental measurements used TIR excitation with s-polarized light. Although a scattered wave is produced in the presence of the antenna, there is no transmitted wave when the bowtie antenna is 
not present. In the FDTD calculation for triangles much smaller than a wavelength, we calculate the scattering efficiency $\left(Q_{\text {scat }}\right)$ as the ratio of scattering cross section $\left(C_{\text {scat }}\right)$ to the metal antenna area $(A)$. We compute the current density distribution in the antennas, use this to compute far field radiated power ( $W_{\text {ext }}$ in Watts), and obtain the scattering cross section $\left(C_{\text {scat }}\right)$ defined by the relation:

$$
C_{\text {scat }}=W_{\text {ext }} / \mathbf{I} \text {, }
$$

where $I=E^{2} / 2 \eta$ is the calculated incident power density $\left(\right.$ Watts $/ \mu \mathrm{m}^{2}$ ) on an antenna with a known current distribution, $E$ is the incident electric field on the antenna, and $\eta$ is the impedance of the medium containing the incident wave.

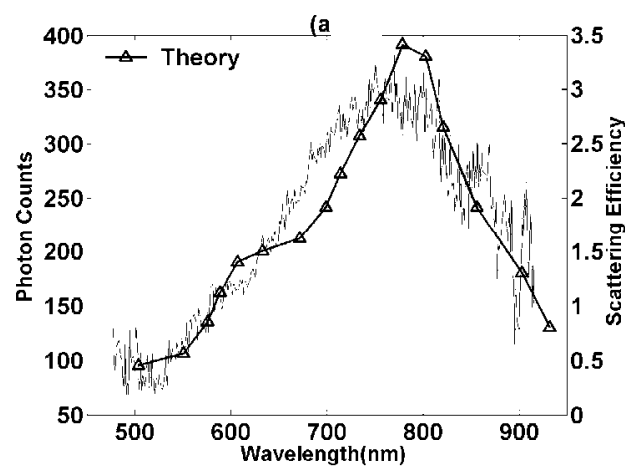

(a)

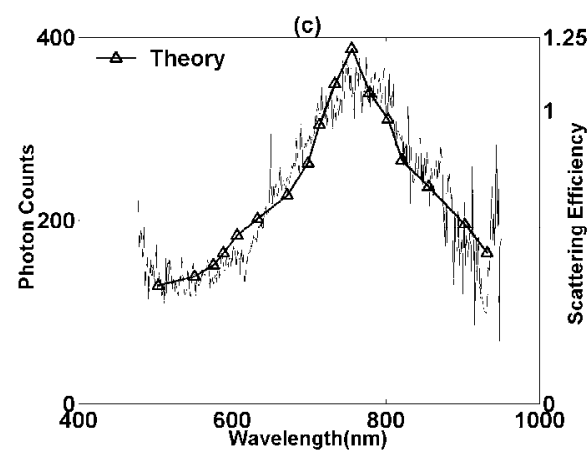

(b)

Figure 3: Plots (a) and (b) compare spectra obtained from FDTD calculations (full curve) and experiments (curve with experimental noise) for different gap lengths. The parameters $\lambda_{\mathrm{rT}}$ and $\lambda_{\mathrm{rE}}$ are the theoretical and experimental peak resonant wavelength with (a) gap $=32 \mathrm{~nm}, \lambda_{\mathrm{rT}}=779 \mathrm{~nm}, \lambda_{\mathrm{rE}} \sim 776 \mathrm{~nm}$ (b) gap $=232 \mathrm{~nm}, \lambda_{\mathrm{rT}}=756 \mathrm{~nm}, \lambda_{\mathrm{rE}} \sim 748 \mathrm{~nm}$.

To calculate $C_{\text {scat }}$, we use antenna theory ${ }^{17}$ to provide a systematic method to compute average radiated power (in watts) from an antenna that has a known current distribution. The total current in the $y$ direction in the metal of the antenna is obtained by integrating the complex polarization current density $\left(\mathrm{J}_{\mathrm{ym}}\right)$ in the metal over $x$ and $z$. The current density in the antenna, the scattering efficiency, and the maximum intensity in the gap are all largest at the same wavelength.

Figures 2 (c) and (d) show the calculated variation in scattering efficiency with wavelength for bowtie antennas of two different gap widths. It will be seen that both the scattering efficiency and intensity in the gap peak at the same resonant wavelength. Similar calculations of scattering efficiency are compared with the experimental results in Figs. 3(a) and (b) for 32nm and 232nm gaps, respectively. To make a fair comparison with the experimental data, the radiated power obtained from the FDTD simulations is multiplied by a constant factor to model the finite acceptance angle of the collection lens (0.8 NA) used in the experiment. The theoretical scattering efficiency at resonance is highest for the smallest gaps $\left(Q_{e x t}=\right.$ 4.1 for a $16 \mathrm{~nm}$ gap), indicating that antennas with smaller gaps radiate more power relative to their area. All the experimental photon counts were scaled to match the peak theoretical FDTD calculated scattered power for a $232 \mathrm{~nm}$ gap in Fig. 3(a) and (b). Because it was not possible to determine the equivalent incident power on the bowtie with TIR excitation, the absolute experimental scattering cross section could not be accurately computed. The experimental scattering spectra shown here rely on the assumption that the incident power is proportional to the measured illumination power from the source as a function of wavelength, scaled by the detection efficiency of our microscope.

The displacement current density, $\mathbf{J}_{D n}=\mathrm{j} \omega \varepsilon_{0} \mathbf{E}_{n}$, in the $x-y$ plane in the gap normal to the edge of the metal is continuous with the current density $\mathbf{J}_{m n}$ normal to the edge inside the metal, where 


$$
\begin{aligned}
& \mathrm{J}_{\mathrm{mn}}=\mathrm{j} \omega \varepsilon_{0}\left(\varepsilon_{\mathrm{r}}-1\right) \mathbf{E}_{\mathrm{mn}}, \\
& (n+j k)^{2}=\varepsilon_{\mathrm{r}}
\end{aligned}
$$

and $\omega$ is the angular frequency, $\varepsilon_{0}$ is the dielectric constant of free space, $\varepsilon_{\mathrm{r}}$ is the relative complex permittivity of the metal, the subscript $n$ stands for the normal component of field, $(n+j k)$ is the complex refractive index of Au taken from Palik et al. ${ }^{15}$, and $\mathbf{E}_{m n}$ is the E field in the metal normal to the edge. The relation between the field components normal to the surface is $\varepsilon_{\mathrm{r}} \mathbf{E}_{m n}=\mathbf{E}_{n}$. Consequently, the enhanced field in the gap is primarily due to the enhanced current density near the tips of the bowtie triangles.

Figure 4 shows the resonant wavelength as a function of gap length. The agreement between theory and experiment is excellent. The bowtie with the smallest gap has the largest peak current density at resonance, and the calculated peak current density decreases as the gap width increases. For a bowtie with a $16 \mathrm{~nm}$ gap width, the peak current density lies at the apex of the triangle, and the current density is fairly uniform with $x$ across the tip region. In this situation, the resonance is at $856 \mathrm{~nm}$ wavelength, where the real part of the dielectric constant of the metal is negative; hence, the impedance of the metal is inductive. Consequently, we might expect to observe a coupled plasmon resonance controlled by the capacity of the gap and the inductance of the tip regions, rather than by the dimensions of the relatively large triangles. The antenna resonance is dominated by the capacitance of the air gap with its associated field in the y-direction for gaps smaller than $60 \mathrm{~nm}$. In this regime, the resonant wavelength decreases with increasing gap (decreasing capacity).

Since the capacity of the air gap is small with a $500 \mathrm{~nm}$ gap, the total current flowing into the gap is small. In this regime, the $E$-field outside the metal is at a maximum in the $z$ direction normal to the plane of the triangles, the coupling between the triangles is decreased, and the current distribution approaches that of a single triangle. For a gap width greater than $60 \mathrm{~nm}$, the field lines near the tip tend to return either to infinity or to the metal triangle from which they left, and the resonant wavelength increases with gap width.

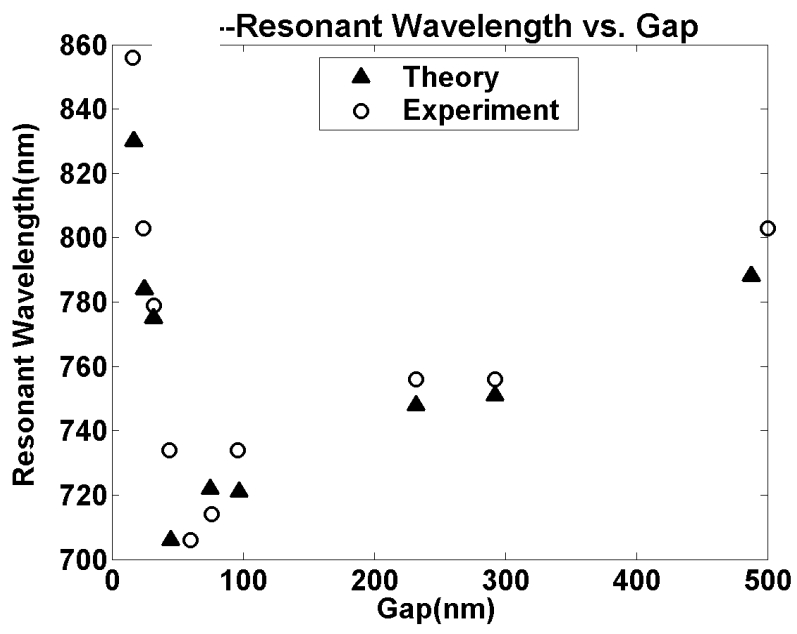

Figure 4: The variation of resonant wavelength with gap for light polarized along the line between the two triangles, with experiment shown as open circles and FDTD simulations as filled triangles. After Ref. ${ }^{12}$. 


\subsection{Two-Photon Effects}

Because the incident pumping field extends over a much larger (diffraction-limited) area and tends to leak into the detector, it is difficult to directly measure the electromagnetic fields in the gap. Instead, we have used two-photon effects that occur only in the region where the optical fields are very strong. Using this approach, we have experimentally determined ${ }^{22}$ the optical intensity enhancement values for the fields in the metal of these structures; these fields closely approximate fields outside the metal near the surface.

Local fields strongly enhanced by the excitation of surface plasmons in rough films, sharp tips, and nanoparticles all give rise to detectable two-photon absorption in $\mathrm{Au}^{18-20}$. The resulting excitation of electrons from the $\mathrm{d}$ valence band to the sp conduction band leads to a broadband emission continuum, termed two-photon-excited photoluminescence (TPPL) in Au. Because of its nonlinear (intensity squared) dependence on excitation intensity, TPPL is a sensitive probe of excitation field strength and distribution. Variations between the TPPL spectra due to differently shaped Au nanoparticles provide evidence for the localized origin of the absorption and subsequent emission ${ }^{21}$. By comparing the strength of TPPL from bowties with TPPL from a smooth Au film ${ }^{22}$, we directly determined absolute values for optical field enhancements of single Au bowties.

A sample-scanning microscope is used to measure the TPPL from individual bowties. A modelocked Ti:sapphire laser producing $120 \mathrm{fs}$ pulses at $\lambda=830 \mathrm{~nm}$ with a repetition rate of $75 \mathrm{MHz}$ was used for excitation, through the epifluorescence port of an inverted optical microscope (Nikon TE300). The value $\lambda=830 \mathrm{~nm}$ was chosen because the smallest gap bowties were measured to be resonant close to this wavelength (see Fig. 4).

The laser is focused through the coverslip to a diffraction-limited spot on the bowtie-air interface using a 1.4 NA, 100X oil objective. Au TPPL is collected by the same objective, and passed through three spectral filters that effectively transmit emissions with wavelengths between $460 \mathrm{~nm}$ and $700 \mathrm{~nm}$ while attenuating $830 \mathrm{~nm}$ laser light with an optical density (OD) $>18$. The luminescence is focused onto a single-photon counting avalanche photodiode (APD) for broadband collection. After optical experiments, the sample is studied with an atomic force microscope (AFM), then coated with a thin Cr layer ( $4 \mathrm{~nm}$ ) for particle analysis in the SEM to carefully measure gap sizes.

The TPPL emission from smooth Au films was used for calibration to directly determine optical field enhancements from bowtie nano-antennas. The optical spectra of the TPPL were identical for the smooth film and the bowties in the collected wavelength range. Figures 5(a) and (b) show that the TPPL is strongly polarization-dependent. Fig. 5(c) shows TPPL from an array of bowties with $20 \mathrm{~nm}$ nominal gap: the inhomogeneity in brightness results from variations in the actual gap width; bowtıes that appear dark were later found by SEM to be shorted. Fig. 5(d) shows an array of $400 \mathrm{~nm}$ gap bowties: both the weaker signal and the ability to optically resolve the two constituent triangles are evident (note the higher pumping power required). Fig. 5(e) is a TPPL image from an Au film square, which required even higher pump power. As expected, TPPL is brightest at the edges, due to the field enhancement caused by localized plasmon resonances at the rough edge produced in the lift-off procedure. Less intense and remarkably uniform TPPL is also observed from the interior of the square. In addition, five hot spots of localized field enhancement are visible with less than one hot spot per $10 \mu \mathrm{m}^{2}$, providing optical evidence of the film's smoothness. 


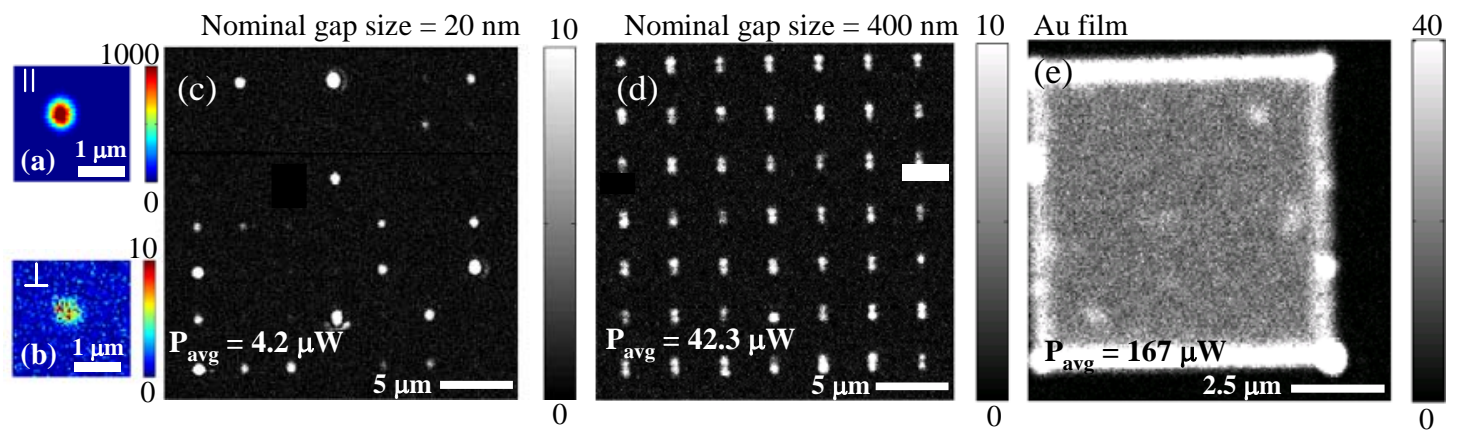

Figure 5: Visible luminescence images from bowtie arrays with nominal gap sizes of $20 \mathrm{~nm}$ (a), (b) and (c), $400 \mathrm{~nm}$ (de), as well from a smooth Au film (e), with $10 \mathrm{~ms}$ per pixel dwell time, $830 \mathrm{~nm}$ pump, and average incident powers (c) $4.2 \mu \mathrm{W}$, (d) $42.3 \mu \mathrm{W}$, (e) $167 \mu \mathrm{W}$. Incident polarization dependence is shown in (a) with E-field in the y-direction and in (b) with E-field in the x direction for a $20 \mathrm{~nm}$ gap bowtie (note different scales). After Ref. [22].

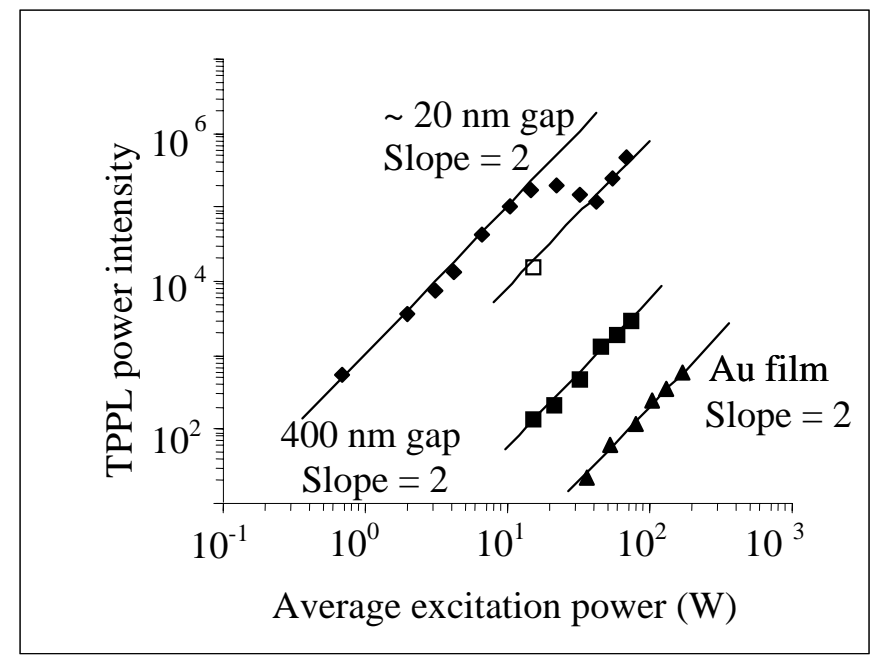

Figure 6: TPPL intensity dependences for a smooth Au film, a $400 \mathrm{~nm}$ gap bowtie, and a bowtie from an array with nominal gap size of $20 \mathrm{~nm}$. Open square: measurement at lower power after the intensity scan. After Ref. ${ }^{22}$, used by permission.

The variation in TPPL intensity with input power is shown in Fig. 6 for a smooth Au film, a single $400 \mathrm{~nm}$ gap bowtie, and a single bowtie from the array with nominal gap size of $20 \mathrm{~nm}$. The APD count rates from the Au film have been normalized for the relative surface area of Au illuminated by the focused laser. For the Au film and $400 \mathrm{~nm}$ gap bowtie, the collected emission shows a quadratic dependence, as expected for TPPL. For the bowtie with a $20 \mathrm{~nm}$ gap, the collected emission follows a quadratic dependence at low average powers. However, as incident power increases, TPPL intensity deviates from this dependence and begins to decrease until, at still higher powers, it again shows a quadratic dependence (but along an $\mathrm{I}^{2}$ curve shifted to the right of the original curve). This behavior occurs for almost all small gap bowties (gaps $<40 \mathrm{~nm}$ ). As is confirmed by SEM observations, when the field intensity becomes large enough to physically damage the tips of the bowtie, the bowtie's field-enhancing capabilities are reduced. Thereafter, the emission follows a new quadratic curve defined by the lower enhancement factor. For subsequent measurements at low powers, the measured TPPL lies on the shifted curve (open 
square in Fig. 6), indicating an irreversible change. Note that the original gap size for this particle is not known since it was damaged before SEM analysis; thus we use the phrase "nominal gap size of $20 \mathrm{~nm}$."

The intensity enhancement in the metal of the bowtie $i, \alpha_{b t}^{i}$, can be calculated from the ratio of TPPL intensities from the bowtie and the film:

$$
\frac{\left\langle T P P L_{b t}^{i}\right\rangle}{\left\langle T P P L_{\text {film }}\right\rangle}=\frac{A_{b t}}{A_{\text {film }}} \times \frac{\left(\alpha_{b t}^{i}\right)^{2}\left\langle P_{b t}^{i}\right\rangle^{2}}{\left\langle P_{\text {film }}\right\rangle^{2}}
$$

where $\left\langle T P P L_{b t}^{i}\right\rangle$ is the (time averaged) TPPL signal when the bowtie $\mathrm{i}$ is centered in the focus of the laser excitation spot, $\left\langle T P P L_{\text {film }}\right\rangle$ is the TPPL signal when the excitation spot is anywhere within the uniform emission region from the Au film, $\left\langle P_{b t}^{i}\right\rangle$ is the average incident power at bowtie i that yields $\left\langle T P P L_{b t}^{i}\right\rangle$, $\left\langle P_{\text {film }}\right\rangle$ is the average incident power at the film that yields $\left\langle T P P L_{\text {film }}\right\rangle, A_{b t}$ is the surface area of the bowtie from which the $\left\langle T P P L_{b t}^{i}\right\rangle$ originates and $A_{\text {film }}$ is total surface area of the Au film from which the $\left\langle T P P L_{\text {film }}\right\rangle$ originates.

We assume the area $A_{\text {film }}$ excited by two-photon absorption of the focused laser is equal to a circular region with a diameter equal to the full width at half maximum (FWHM) of the square of the intensity point spread function (PSF) of our microscope objective. For $\lambda=830 \mathrm{~nm}$ and NA $=1.4$, the measured FWHM from a diffraction-limited TPPL spot is equal to $214 \mathrm{~nm}$; thus, $A_{\text {film }}=35600 \mathrm{~nm}^{2}$. FDTD calculations show that plasmonic current densities and optical near-field intensities are concentrated in a confined region of each bowtie, e.g., within the Au nearest the gap, particularly for bowties with small gap sizes. Thus $A_{b t}$ is also gap-dependent, and must be calculated.

As mentioned above, the strongest optical fields are found in the gap between the constituent triangles. However, the experimentally observed TPPL certainly arises from electromagnetic fields in the metal. Therefore, the intensity of the TPPL should be proportional to the fourth power of electric field in the metal, $E_{m}$, and the total TPPL power will be correlated to the integral of $\left|E_{m}\right|^{4}$. For comparison with these experimental results, plane wave excitation normally incident on the bowties is assumed in FDTD calculations. For an incident electric field amplitude $E_{0}$, the tangential electric field at the metal surface is $E_{\text {inc }}=2 E_{0} /[1+(n+j k)]$. In the metal film, the electric field falls off as exp(-kz). FDTD simulations for the bowties show that, to a good approximation, the E-field in the bowties also falls off as exp(-kz). Since all fields have the same z-dependence in the metal, the integral of $\left|E_{m}\right|^{4}$ needs to be performed only along the $\mathrm{x}$ and $\mathrm{y}$ directions, just below the surface of the metal in the FDTD computations

We employed FDTD calculations to determine the square of the intensity enhancement in the bowtie i, $\left|a_{b t}^{i, F D T D}\right|^{2}$ using

$$
\left|\alpha_{b t}^{i, F D T D}\right|^{2}=\frac{\iint\left|E_{b t, m}\right|^{4} d x d y}{\iint E_{\text {incident }}^{4} d x d y}
$$

where $\left|E_{b t, m}\right|^{4}=\left(E_{b t, m x}^{2}+E_{b t, m y}^{2}+E_{b t, m z}^{2}\right)^{2}$, and the integrals extend over the area of the bowtie. The experimental and theoretical results for $\left|\alpha_{b t}^{i}\right|^{2}$ are shown in Fig. 7 for various gap widths. The effective area $A_{b t}$ of the TPPL source is determined from the following integral: 


$$
A_{b t}=\int E_{m}^{4} d x d y / E_{\max }^{4}
$$

where $E_{\max }$ is the maximum field in the bowtie. $A_{b t}$ is a function of gap size, increasing as gap width increases. The theoretical $A_{b t}$ for the smallest gap width $(16 \mathrm{~nm})$ was $642 \mathrm{~nm}^{2}$, indicating that the field was confined to approximately one-tenth of the $\sim 6530 \mathrm{~nm}^{2}$ area of the metal in the bowtie. Combining these values for $A_{b t}$ in Eq. (1) with the experimentally determined TPPL intensities yields $E^{2}$ enhancement factors of greater than $10^{3}$, or greater than $10^{6}$ for $\alpha^{2}$ for bowties with the smallest gaps (Fig. 7). These are the largest such factors reported to date for lithographically produced nanoantennas.

The $E^{4}$ values for theory and experiment were in good agreement for gap widths less than 30nm, but not as good for gap widths of $40 \mathrm{~nm}-60 \mathrm{~nm}$. For nominal gap widths of $400 \mathrm{~nm}$, there was good agreement again. This difference can be explained by the fact that the plane wave excitation used for FDTD closely approximates the experimental conditions for small gap bowties, but does not accurately model intermediate gaps where the triangles are not uniformly pumped by the focused laser spot. To avoid this difficulty for gap widths of $400 \mathrm{~nm}$, we excited and collected TPPL from each triangle of the bowtie separately and summed the two, since the coupling is minimal. Furthermore, since a bowtie with a $400 \mathrm{~nm}$ gap was too large to simulate, the $E^{4}$ enhancement value was obtained by doubling the value from FDTD simulations for a single triangle (Fig. 7, triangle).

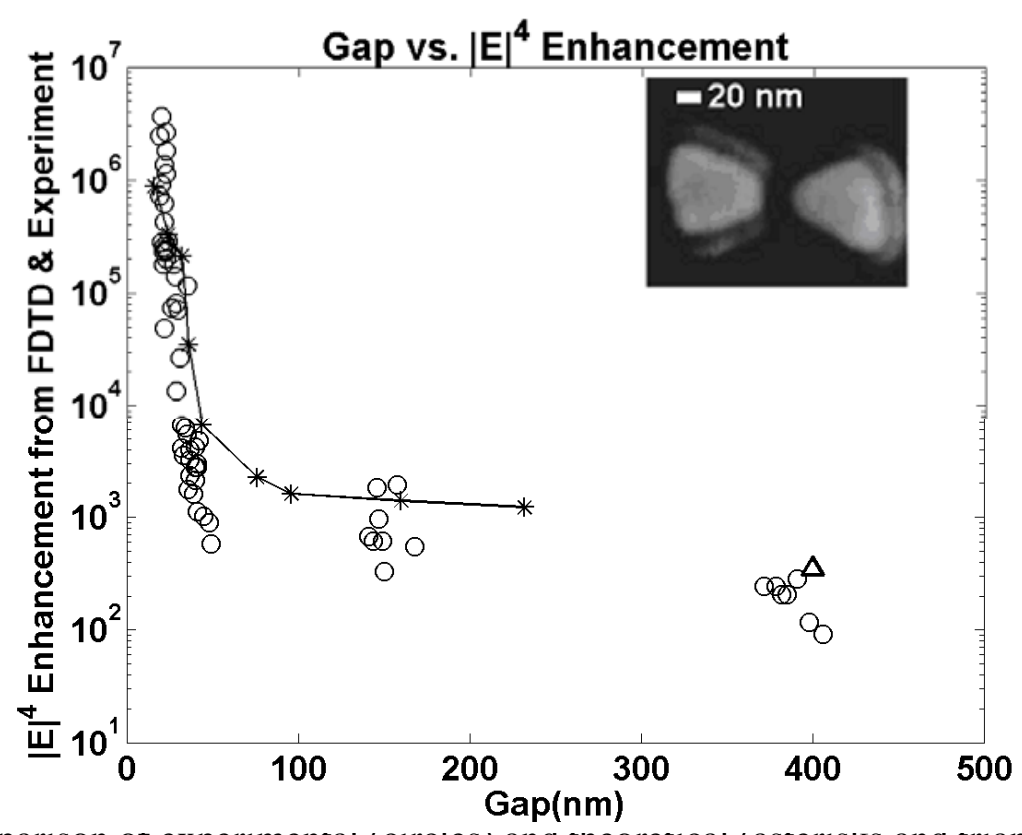

Figure 7: Comparıson or experımentaı (cırcıes) ana ıneoreucaı (asterısks ana triangı) values of the square of intensity enhancement, $\left(\alpha_{b t}{ }^{i}\right)^{2}$, for bowties with gaps of $16 \mathrm{~nm}$ to $406 \mathrm{~nm}$. FDTD simulations for $\left(\alpha_{b t}{ }^{i}\right)^{2}$ from an individual triangle are used for comparison to the bowties with nominal gap size of 400 $\mathrm{nm}$. Excellent agreement is observed, especially for the largest and smallest gap sizes, where experimental conditions most closely approximate the theoretical treatment.

\section{Rahman Effect}

Recent progress in a separate research area, surface-enhanced Raman scattering (SERS), is quite relevant to the issue of electromagnetic enhancement and measurement of the properties of nearby molecules. Reports of SERS from single molecules in/near/between colloidal metal particles requiring enhancements as large as $10^{14}$ has led to much speculation about the mechanism, which is attributed partly to electromagnetic enhancements and partly to chemical interactions between the molecule and the metal. A key difficulty in the further use of SERS has been the lack of control of the "special sites" that give 
huge signals from the inhomogeneous array of metal particles in aqueous sols, due to the inability to controllably produce closely spaced pairs of small metal spheres. In contrast, our lithographically prepared metallic bowties provide a unique and controllable configuration of two closely spaced metallic objects.

In our recent work, we have observed Raman scattering spectra (as shown in Fig. 8) of p-mercaptoaniline (pMA) molecules bonded via S-Au bonds to the surface of a single Au bowtie, with representative spectra shown in the figure. Note that these data were acquired with a simple, low power cw semiconductor diode laser! Surprisingly, although many molecules are bonded to the Au, the spectra fluctuate and change with time in a fashion similar to the single-molecule SERS reports, which suggests changes in the charge states of the molecules. These results demonstrate our ability to use our fabrication, spectroscopic, and modeling skills to derive insight into the actual mechanism of the single-molecule Raman observations.

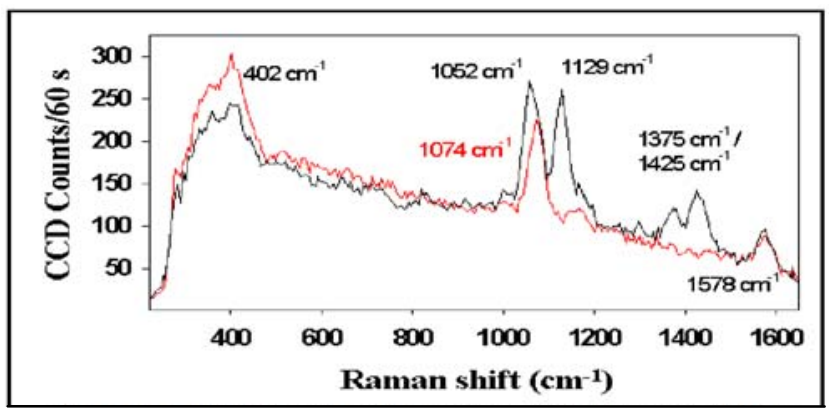

Figure 8. Raman scattering spectra of p-mercaptoaniline molecules bonded via S-Au bonds to the surface of a single Au bowtie.

We have acquired preliminary data using nanoscale Au bowties as controlled, coupled particles to enhance the SERS signal of molecules attached through a thiol linkage. Recent experiments by the Moerner lab have quantified the local electromagnetic enhancement of bowties pumped at their resonant frequency; small thiol molecules enable controllable deposition of the molecules at a known coverage, which should enable the determination of the nature of the SERS enhancement. Specifically, we are using pMA as a model SERS molecule. A single-molecule confocal microscope, equipped with a beamsplitter sending $90 \%$ of the light to a grating spectrometer coupled to a CCD camera, is used to obtain Raman spectra. The remaining $10 \%$ of the light is sent to a single photon counting module to collect broadband Raman information on the $10 \mathrm{~ms}$ (or faster) timescale. A diode laser at $830 \mathrm{~nm}$ is used to resonantly pump $20 \mathrm{~nm}$ gap bowties that have been incubated in a solution of pMA to provide a monolayer on the Au particle, thus locating several thousand molecules near the bowtie gap.

Three representative spectra from the same bowtie are shown in Fig.9. Each spectrum has a $2 \mathrm{~s}$ integration time, taken after 0,108 , and 228 seconds of continuous illumination. The most notable feature of the SERS spectra is their dramatic fluctuation for pMA on Au in both overall intensity as well as the Raman modes. At $0 \mathrm{sec}$, three modes (at 390, 1078, and $1590 \mathrm{~cm}^{-1}$ ) are present that can be assigned to known Raman modes of bulk pMA ${ }^{4}$. The SERS spectrum changes significantly, as seen in the exposure taken after $108 \mathrm{~s}$ of illumination. Several new lines now appear at 1177, 1351, and $1437 \mathrm{~cm}^{-1}$, and their peak assignments are consistent with previous SERS experiments on roughened Ag films. The authors suggest that these new modes are a signature of electron transfer from the Fermi level of the Ag to the pMA molecule; it is conceivable that a similar charge transfer effect is the cause of the observed enhancement of Raman lines mentioned above. Although the Raman spectra changed significantly during our experiment, the appearance of the new charge transfer modes was always accompanied by an overall 
intensity increase; in the exposure taken after $228 \mathrm{~s}$, a chemical change of the pMA-Au system is producing a large, temporary SERS enhancement.

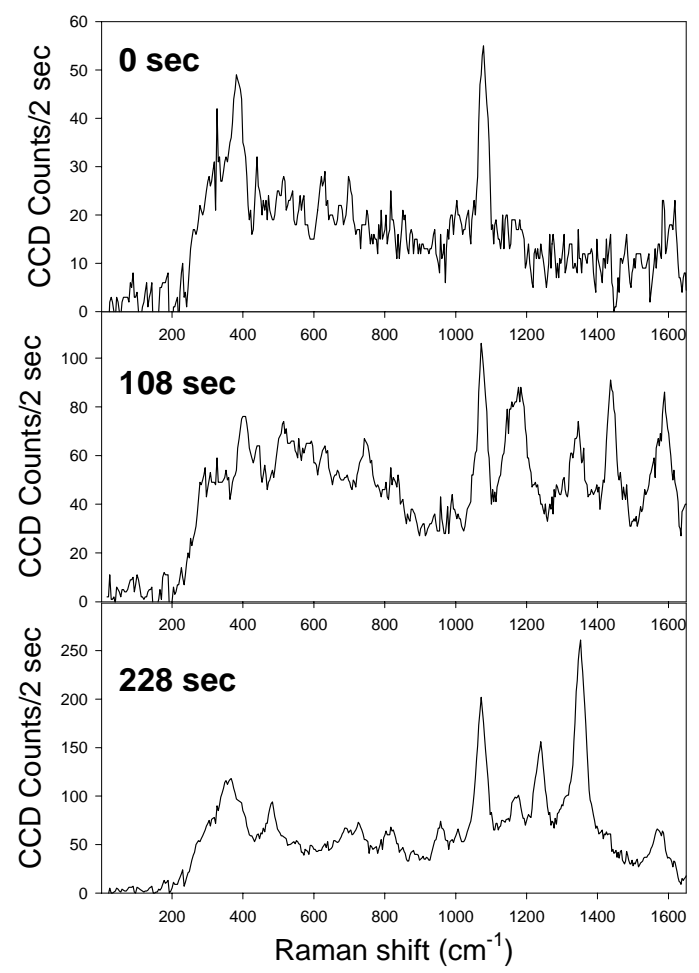

Figure 9. Raman scattering showing fluctuations in the spectra with time.

\section{Conclusions}

Using e-beam lithography, we have fabricated Au bowtie nano antennas that have resonances in the visible/near-ir wavelength range. FDTD calculations have been used to predict resonance frequencies, field profiles, and local field enhancements. Experimental measurements by TIR microscopy of peak scattering wavelength for bowties with a range of gap sizes from $20 \mathrm{~nm}$ to $500 \mathrm{~nm}$ are in good agreement with theory. Using TPPL, we have also experimentally measured optical intensity enhancements at $\mathrm{Au}$ bowtie nanoantennas of various gap sizes, and find good agreement with FDTD simulations. For small gap bowties, the field intensity enhancement is $>10^{3}$ confined to a region $\sim 650 \mathrm{~nm}^{2}$, which may be interpreted as a dramatic improvement in the mismatch between conventional optical excitations and nanoscale structures. With optimization of the fabrication, we expect Au bowties will be reproducibly manufactured, either individually on a scanning probe or in large arrays on a single substrate. The large enhanced fields in the metal will also lead to similarly enhanced, localized fields on the metallic surface and inbetween the two bowties. This design will yield extremely intense near-field optical light sources with high local contrast, enabling applications ranging from the elucidation of SERS mechanisms, ultrasensitive biological detection, single-molecule spectroscopy, and nanometer-scale lithography, to highresolution optical microscopy and spectroscopy.

We are currently pursuing our initial investigation of Raman scattering using bowtie excitation. We feel that controllable experiments with bowtie excitation should give us much more insight into the Ra- 
man excitation mechanisms. We are also using the bowties to obtain two-photon exposure of photoresist to obtain high definition images. We are also using our metal deposition techniques to deposit bowties on the end of a flattened AFM tip to make a near-field scanning optical microscope with definition of less than $30 \mathrm{~nm}$ and good efficiency.

\section{Acknowledgements}

This work was supported partly by the U. S. Department of Energy Grant No. DE-FG03-00ER45815 and by the National Institutes of Health Grant No.GM65331-R21. The deposition work, manufacture of the bowties, and theoretical analysis using FDTD techniques was carried out by A. Sundaramurthy, who was supported on our DOE grant; the TPPL and Raman measurements were carried out by D. Fromm and J. Schuck, in cooperation with Sundaramurthy, and supported on our NIH grant. We thank Prof. Andy Neureuther, UC Berkeley, for use of TEMPEST 6.0 FDTD software developed by his group.

\section{References}

1. E. Betzig, J.K. Trautman, T.D. Harris, J.S. Weiner, and R.L. Kostelak, Science 251, 146 (1991).

2. F. Zenhausern, Y. Martin, and H.K. Wickramasinghe, Science 269, 1083 (1995).

3. J.L. Bohn, D.J. Nesbitt, A. Gallagher, J. Opt. Soc. Amer. A 18, 2998-3006 (2001).

4. L. Novotny, E.J. Sanchez, and X.S. Xie, Ultramicroscopy 71, 21 (1998).

5. A. Hartschuch, E.J. Sanchez, X.S. Xie, and L.Novotny, Phys. Rev. Lett. 90, 095503 (2003).

6. S.A. Maier, P.G. Kik, and H.A. Atwater, Appl. Phys. Lett. 81, 1714(2002).

7. K.B. Crozier, A. Sundaramurthy, G.S. Kino, and C.F. Quate, J. Appl. Phys. 94, 4632 (2003).

8. D.A. Genov, A.K. Sarychev, V.M. Shalaev, and A. Wei, Nano Lett. 4, 0343710 (2004).

9. E. Hao and G.C. Schatz, J. Chem. Phys. 120, 357 (2004).

10. W. Rechberger, A.Hohenau, A. Leitner, J.R. Krenn, B. Lamprecht, and F.R. Ausenegg, Opt. Commun. 220, 137 (2003).

11. R.D. Grober, R.J. Schoelkopf, and D.E. Prober, Appl. Phys. Lett. 70, 1354(1997).

12. D.P. Fromm, A. Sundaramurthy, P. J. Schuck, G. Kino and W.E. Moerner, Nano Lett. 4, 957 (2004).

13. J.G. Goodberlet, J.T. Hastings, H. I. Smith, J. Vac. Sci. Technol. B 19, 2499 (2001).

14. TEMPEST 6.0, Electronics Research Laboratory, University of California, Berkeley, California.

15. E.D. Palik, Handbook of Optical Constants (Academic Press, Orlando, Florida, 1985).

16. A. Sundaramurthy, P.J. Schuck, D.P. Fromm, W.E. Moerner and G. Kino, unpublished work, submitted to Phys. Rev. B.

17. S. Ramo, J.R. Whinnery and T. Van Duzer, Fields and Waves in Communication Electronics, $2^{\text {nd }}$ Ed. (Wiley, New York, 1984) pp. 586-589.

18. C. K. Chen, A. R. B. de Castro, and Y. R. Shen, Phys. Rev. Lett. 46, 145 (1981).

19. G. T. Boyd, Z. H. Yu, and Y. R. Shen, Phys. Rev. B 33, 7923 (1986).

20. M. R. Beversluis, A. Bouhelier, and L. Novotny, Phys. Rev. B 68, 115433 (2003).

21. A. Bouhelier, M. R. Beversluis, and L. Novotny, Appl. Phys. Lett. 83, 5041 (2003).

22. P.J. Schuck, D.P. Fromm, A. Sundaramurthy, G.S. Kino and W.E. Moerner, Phys. Rev. Lett. 94, 017402 (2005).

\section{Patents}

D.A. Fletcher and G.S. Kino, "Collection Mode Lens System" US patent number 6,477,298 (issued $11 / 5 / 02)$. 


\section{$\underline{\text { Publications }}$}

1. D.A. Fletcher, D. Simanovskii, D.V. Palanker, K.B. Crozier, C.F. Quate, G.S. Kino, and K.E. Goodson, "Microfabricated Solid Immersion Lens with Metal Aperture," Proceedings of the IEEE/LEOS International Conference on Optical MEMS, Kauai, Hawaii, (2000)

2. D.A. Fletcher, D. Simanovskii, D. Palanker, K.B. Crozier, C.F. Quate, G.S. Kino, K.E. Goodson, "Microfabricated solid immersion lens with metal aperture," Conference: 2000 IEEE/LEOS International Conference on Optical MEMS, 21-24 Aug. 2000, Kauai, HI, US

3. K.B. Crozier, D.A. Fletcher, G.S. Kino, C.F. Quate, "Micromachined silicon nitride solid immersion lenses," Conference: 2000 IEEE/LEOS International Conference on Optical MEMS, 21-24 Aug. 2000, Kauai, HI, USA

4. D.A. Fletcher, K.B. Crozier, G.S. Kino, C.F. Quate, and K.E. Goodson, "Micromachined Scanning Refractive Lens," Proceedings of the Solid-State Sensor and Actuator Workshop, Hilton Head, SC, (2000)

5. D.A. Fletcher, K.B. Crozier, K.E. Goodson, C.F. Quate, G.S. Kino," Optical characterization of microfabricated solid immersion lenses," Proceedings of the SPIE - The International Society for Optical Engineering; 3919, 100-108 (2000)

6. D.A. Fletcher, K.B. Crozier, C. F. Quate, G.S. Kino, K.E. Goodson, D. Simanovskii, D. V. Palanker, "Near-field infrared imaging with a microfabricated solid immersion lens," Applied Physics Letters, 77 (14), 2109-2111 (2000)

7. D.A. Fletcher, K.E. Goodson, G. S. Kino, "Focusing in microlenses close to a wavelength in diameter" Optics Letters, 26, 399-401 (2001)

8. D.A. Fletcher, K.B. Crozier, C. F. Quate, G. S. Kino, K.E. Goodson, D. Simanovskii, D. V. Palanker, "Refraction contrast imaging with a scanning microlens," Applied Physics Letters, 78, pp. 35893591, (2001)

9. D.A. Fletcher, N.U. Webb, G.S. Kino, C.F. Quate, and K.E. Goodson, "Thermal Microscopy with a microfabricated Solid Immersion Lens," Proceedings of the IEEE/LEOS International Conference on Optical MEMS, Okinawa, Japan, (2001)

10. D.A. Fletcher, D.V. Palanker, P. Huie, G.S. Kino, and T.I. Smith, "Microfabricated Solid Immersion Lens for Infrared Microspectroscopy," Proceedings of the International Workshop on Microspectroscopy, Porquerolles Island, France, (2001)

11. K. B. Crozier, A. Sundaramurthy, D.A. Fletcher, G. S. Kino, C. F. Quate, "Nearfield Optics with solid immersion lenses and sharp metal probes," 2001 First IEEE Conf. On Nanotechnology, IEEENANO 2001, 28-30 October 2001, Maui, HI 
12. D. A. Fletcher, K.B. Crozier, K.W. Guarini, S. C. Minne, G. S. Kino, C. F. Quate, K.E. Goodson, "Microfabricated silicon solid immersion lens," Journal of Microelectromechanical Systems; vol.10, 450-459 (2001)

13. K.B. Crozier, A. Sundaramurthy, C.F. Quate and G.S. Kino, "Nearfield Optics with Solid Immersion Lens and Sharp Metal Probes,” Nanotechnology, 2001. IEEE-NANO 2001 Proceedings of the 2001 1st IEEE Conference, pp. 501-506 (28-30 Oct. 2001)

14. K.B. Crozier, D. A. Fletcher, G.S. Kino, C.F. Quate, "Micromachined silicon nitride solid immersion lens," Journal of Microelectromechanical Systems, vol. 11, pp. 470 -478 (2002)

15. G.S. Kino, A. Sundaramurthy, K.B. Crozier, D. Fromm, D. Fletcher, W.E. Moerner, C.F. Quate and K. Goodson, "Optical Antennas for Microscopy of Nanostructures," Proceedings of the 20th Symposium on Energy Engineering Sciences, Argonne National Laboratory, Argonne, IL, pp. 99-106 (May 20-21, 2002

16. Crozier, KB; Sundaramurthy, A; Kino, GS; Quate, CF, Fromm, DP, Moerner, WE, "Field enhancement and resonance in optical antennas" presented at Trends and Optics and Photonics Series: QELS, Baltimore, MD; QELS Tech. Digest Series: 2003, vol. 89, pp. QTUD3/1-QTUD3/2 (6/16/6/2003)

17. Crozier, KB; Sundaramurthy, A; Kino, GS; Quate, CF, "Optical antennas: resonators for local field enhancement," Journal of Applied Physics, vol. 94, no. 7, pp. 4632-4642 (1 Oct 2003)

18. D.P. Fromm, A. Sundaramurthy, P. J. Schuck, G.S. Kino, and W.E. Moerner, " Gap-dependent Optical Coupling of Single 'Bowtie' Nanoantennas Resonant in the Visible," Nano Letters, Vol. 4, pp. 957-961 (March 8, 2004)

19. P.J. Schuck, D.P. Fromm, A. Sundaramurthy, G.S. Kino, W.E. Moerner, "Improving the mismatch between light and nanoscale objects with gold 'bowtie' nano-antennas," Phys. Rev. Letters, Vol. 94, pp. 017402 (2005)

20. G. S. Kino, Arvind Sundaramurthy, P.J. Schuck, D.P. Fromm, and W.E. Moerner, Optical Field Enhancement with plasmon resonant bowtie nanoantennas, Ed. M. Brongersma, Kluwer Academic/Plenum Publishers, invited chapter to be published 2005 